\title{
Literale Praktiken und literale Kompetenz
}

\begin{abstract}
Die linguistische Fundierung des Praktikenkonzepts kann von der starken Stellung des Konzepts in der Literalitätsforschung profitieren. Hier spielt der Begriff seit Beginn der kontroversen Diskussion zu den „Konsequenzen der Literalität“ ab Beginn der 1980er Jahre eine zentrale Rolle. Die Forschung zeigt, dass „literacy practices“ als institutionell und kontextuell gebundene Formen des schriftlichen Sprachgebrauchs die pragmatischen Transmissionsriemen für den Aufbau literaler Kompetenz sind. An drei empirischen Bereichen, dem Erwerb von Textsortenwissen vor Beginn des eigenen Schreibens (protoliteraler Erwerb), dem Erwerb von phonologischer Bewusstheit und von Prozeduren des Textreferierens (schulischer Erwerb) und schließlich dem Erwerb von intertextuellen Praktiken wissenschaftlichen Zitierens (wissenschaftlicher Erwerb) diskutiert der Beitrag exemplarisch Leistungsmöglichkeiten und Grenzen praktikentheoretischer Konzepte für die Erklärung des beobachtbaren Sprachlernens.
\end{abstract}

\section{Einführung - Warum „Praktiken“?}

Wer einen Friseursalon betreten hat, der kann - selbst, wenn sich das Personal freundlich an ihn wendet - keine Peking-Ente mehr bestellen. Und wer in der Wissenschaft reüssieren möchte, der kann - selbst wenn sie ihm persönlich näher liegen mögen - dort nicht mit Stammtischweisheiten hausieren gehen. Sprecherinnen und Sprecher, Schreiberinnen und Schreiber werden frei geboren, aber sie agieren im Kontext von kulturellen Handlungsfeldern und daran gebundenen Praktiken, und es ist die Übereinstimmung mit diesen Praktiken, die den Sinn ihres Handelns sichert.

Jedes Zeichen - so hat Karl Bühler in seiner Sprachtheorie (1982) gefordert sei sub specie einer menschlichen Handlung zu analysieren. Und - so könnte man, das Diktum erweiternd, fortfahren - sprachliche Handlungen sind häufig mit Gewinn sub specie einer Praktik zu analysieren, der sie zugeordnet werden können und die ihren sozialen Sinn bestimmt. Vielleicht gilt dies noch einmal verstärkt für den Schriftgebrauch. Auch Schreiber und Leser agieren im Kontext von kulturellen Handlungsfeldern und daran gebundenen Praktiken, die sozial typisiert, wertbesetzt und sprachlich wie kommunikativ in hohem Maße normgebunden sind. 


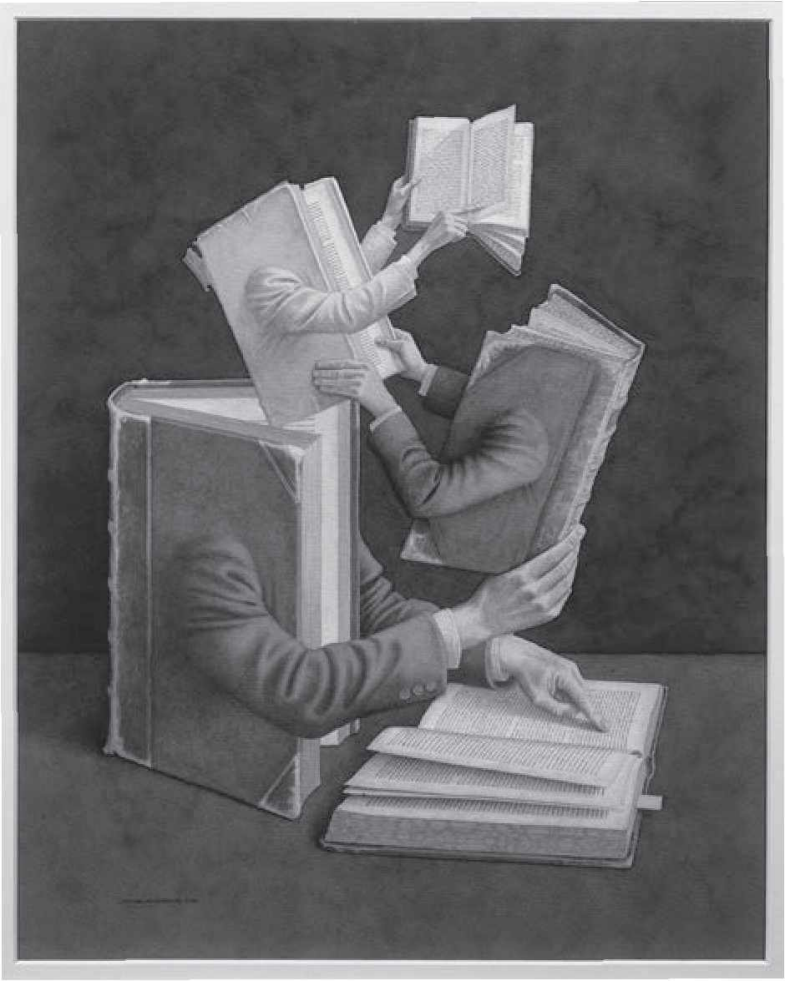

Abb. 1: Jonathan Wolstenholme: „Cross References“ (2003)

Ein Beispiel: Das Bild, das Sie in Abbildung 1 sehen, stammt von dem englischen Grafiker Jonathan Wolstenholme. Es trägt den Titel „Cross References“ (2003), „Querverweise“. Querverweise sind zunächst routinehaft lexikalisierte Zeichen (z.B. ein Partizip wie das ,obengenannte‘ Bild). Sie bilden sich als eine sprachliche Form der Intertextualität historisch ab dem 16. Jahrhundert heraus und haben dabei auch viele Wandlungen erfahren (vgl. Gloning 2003, S. 405 ff.). Es geht bei den Querverweisen nicht nur um sprachliche Zeichen, auch nicht um einzelne Verweishandlungen. Zugrunde liegt vielmehr eine rekurrente literale Praxis des Verfolgens intra- und intertextueller Verweise. $\mathrm{Zu}$ ihr zählt der ganze Gebrauchszusammenhang. Die Leser sind Büchermenschen, es sind Typen, die lesen. Die Akteure sind sozial typisiert. Ebenso ist es mit der Handlung selbst: Die Leser folgen den Verweisen und das Lesen geschieht in mehreren Texten zeitlich parallel, eben ,quer zur Sukzession des einzelnen linearen Textes. Die soziale Typisierung der Handelnden und der äußeren Form des Handelns sowie der damit verbundenen Motive und Erwartungen macht aus der je individuellen 
Praxis eine soziale Praktik. ${ }^{1}$ Es ist diese Musterhaftigkeit des gezeigten Verhaltens, die „das ganze System beschwört, zu dem sie gehört“ (Bourdieu 1987, S. 128). In der Praktik kommt ein Habitus im Sinne Bourdieus zum Ausdruck, sie verweist pars pro toto (vgl. ebd.) als Segment auf eine ganze Kultur des Umgangs mit Texten.

Die Praktik bezieht freilich auch die Schreiber ein, die aufeinander verweisen und ihr daher zuzurechnen sind. Dabei geht es auch nicht nur um das Verweisen. Es geht um einen - genuin literalen - Typus des Umgangs mit Texten und ihrer Intertextualität. Er ist dem fachlichen Umgang mit Texten, dem Recht und der Theologie, im Weiteren der Domäne der Wissenschaft zugeordnet, und eng mit bestimmten Werten verbunden, z.B. Intersubjektivität und Überprüfbarkeit, die dort hochgeschätzt sind. Wie in den verschiedenen Schalen einer Zwiebel verbinden sich in der Praktik nach dem Form-in-Form-Prinzip die Schichten von der sozialen Form des Handelns bis zum sprachlichen Strukturelement. Auf das Beispiel der Praktiken wissenschaftlicher Intertextualität werde ich auch am Ende meines Beitrags zurückkommen.

Einige Worte zum Aufbau des Beitrags: Nach einer kurzen fachgeschichtlichen Hinführung zum Thema stellen der dritte bis fünfte Teil des Beitrags Zusammenhänge von literaler Kompetenz und literalen Praktiken an drei Beobachtungsfeldern vor: vorschulische protoliterale Praktiken, schulische Schriftpraktiken und schließlich wissenschaftlich literale Praktiken. Die Gliederung folgt damit einerseits möglichen Etappen einer literalen Spracherwerbsgeschichte. Andererseits erscheint das Praktikenkonzept in der Anwendung auf diese Etappen jeweils in einem anderen Licht.

Im Sinn der in der Einleitung zu diesem Band vorgestellten Dreigliederung in superstrukturelle, makrostrukturelle und mikrostrukturelle Aspekte kommunikativer und sprachlicher Praktiken geht es dabei um folgende Fragen:

1. Superstruktureller Aspekt: Aus welchen, im weiteren Sinn sozial und historisch situierten Praxiskonstellationen resultieren bestimmte Formen des Umgangs mit Schrift, mit Texten und geschriebener Sprache?

2. Makrostruktureller Aspekt: Welche Rolle spielen literale Artefakte (das Alphabet, didaktische Gattungen) und methodische Praktiken als koordinierte Handlungsgefüge für den Aufbau literaler Kompetenz? ${ }^{2}$ Der Aspekt steht im Abschnitt 4 über die schulischen literalen Praktiken im Vordergrund.

1 Vgl. hierzu die bei Berger/Luckmann (1980, S. 49-138) dargestellten Zusammenhänge von Habitualisierung, Institutionalisierung und sozialer Objektivierung.

2 In der soziologischen Theorie sozialer Praktiken wird der Artefaktbegriff häufig lediglich auf materielle und technische „Gegenstände“ beschränkt. In der Theorie literaler Praktiken werden 
3. Mikrostruktureller Aspekt: Welche Formen und Prozeduren der Handlungssequenzierung und -indizierung werden dadurch sprachlich hervorgebracht? Dieser Aspekt wird vor allem im Abschnitt 5 zu literalen Praktiken der Wissenschaft herausgestellt.

\section{Woher kommt das Interesse an literalen Praktiken?}

Unter ,literacy‘ wird im Englischen gewöhnlich die Fähigkeit verstanden, Schriftzeichen zu lesen und zu schreiben, das heißt, geschriebene Sprache zu dekodieren bzw. Sprache schriftlich enkodieren zu können. Diese Kulturtechnik „literacy“ und die Frage nach ihren kulturellen und kognitiven Folgen verbindet sich in der Forschung zu Schriftlichkeit und Schreiben bis in die 1980er Jahre mit der sogenannten „starken Literalitätshypothese“ (vgl. zur Diskussion Brockmeier 1997; Sterponi/Lai 2013): Danach ist die Fähigkeit zu logischer Analyse und Komponentenzerlegung, etwa von Texten, sowie zu den korrespondierenden Kompositionen und Konstruktionen, etwa in philosophischen Systemen der griechischen Antike, eine direkte Konsequenz des Umgangs mit dem Kompositionssystem einer Alphabetschrift (vgl. Goody/Watt/Gough 1986; Stetter 1997). Sie begründet nach dieser These eine historische Technologie des Sprachdenkens, was sich noch in einem Buchtitel wie W.J. Ongs (1987) „Oralität und Literalität. Die Technologisierung des Wortes" spiegelt.

Die Prominenz der Kulturtechnik Schrift und die ihr zugeschriebenen „Konsequenzen“ für Denken und Lernen haben lange den Blick auf die Tatsache verstellt, dass Techniken nur abhängig von den Praktiken funktionieren, in die sie eingebunden sind bzw. eingebunden werden. Bekannt ist etwa die Tatsache, dass das Alphabet schon Jahrhunderte existierte, bevor man auf die Idee kam, es als Ordnungsprinzip für Nachschlagewerke zu nutzen. Illich (1991), Parkes (1999) und Saenger (1999) haben den hier zugrundeliegenden Praktikenwandel im Spätmittelalter sehr gut beschrieben. Sie zeigen, wie historisch-kulturell eine Situation entsteht, in der die bedeutungsbezogene Textanalyse wichtiger wird als zum Beispiel das kalligraphische Kopieren oder gottesdienstliche Rezitieren alpha-

ganz dezidiert auch semiotische Vergegenständlichungen darunter gefasst, die dann oft ihrerseits wieder als didaktische Artefakte in Lehrmaterialien vergegenständlicht werden, z.B. Alphabetbücher, Anlauttabellen, Textmuster und Mustertexte usw. 
betschriftlicher Seiten. Hier treten nicht Schriftsysteme und Schrifttechniken, sondern zuallererst Praktiken des Schriftgebrauchs in Opposition zueinander. Florian Coulmas schreibt entsprechend schon 1981: „Schriftsysteme sind nicht durch die explizite Definition ihrer Elemente, sondern durch die Praxis ihres Gebrauchs gegeben“ (Coulmas 1981, S. 30). Diese literalen Praktiken und die mit ihnen verbundenen sozialen, emotionalen, kognitiven und sprachlichen Einstellungen, Interessen und Verhaltensmuster prägen den Schriftgebrauch und entscheiden damit auch über die Konsequenzen der Literalität.

Im Fachdiskurs zum Durchbruch kam das Konzept der ,literacy practices‘ Ende der 1970er Jahre mit den Untersuchungen der Psychologen Scribner und Cole. Sie stellten 1978 in einer vielzitierten Studie zum mehrschriftigen Sprachgebrauch des Stammes der Vai in Liberia die bis dahin weithin akzeptierte starke Literalitätshypothese in Frage. Die Vai verwenden die Schrift in drei unterschiedlichen institutionellen und sprachlichen Kontexten: Arabisch in religiösen Kontexten, Englisch in der Schule und Vai, das eine Mitte des 19. Jahrhunderts eingeführte eigenständige Silbenschrift hat, im Alltag. Die Autoren konnten nachweisen, dass die text- und satzanalytischen und sprachlich-logischen Denkformen nur im schulischen Kontext und vor dem Hintergrund der schulischen Erwartungen an den Schriftgebrauch belegbar waren. Die säkulare Schule entwickelt und kultiviert einen Typ des rezeptiven und produktiven Umgangs mit Texten, der auf Text- und Sprachanalyse, auf eine situationsentbundene Sprachlichkeit und eine textbezogene semantische Kohärenz zielt (vgl. Scribner/Cole 1978, S. 457). Dies aber kann in anderen Praktikenzusammenhängen ganz anders aussehen: Nicht das Medium, die Praktik bestimmt die Botschaft (vgl. für einen Überblick HäckiBuhofer 2000).

\section{Protoliterale Praktiken}

Protoliterale Praktiken sind Praktiken des Schriftgebrauchs vor Beginn des eigentlichen Schriftspracherwerbs, also bevor Kinder selbst im Medium geschriebener Sprache zu lesen und schreiben beginnen (vgl. Feilke 2003, S. 178 f.). So können Kinder dadurch, dass vorgelesen wird, Schrifttexte rezipieren; und sie können, schon bevor sie selbst schreiben können, anderen Texte diktieren. Auch wenn der eigene Name gemalt, aber nicht eigentlich geschrieben wird, haben wir es mit einer protoliteralen Tätigkeit zu tun. Besonders interessant ist es, wenn Kinder protoliteral Lesen \& Schreiben spielen. Ein solches Beispiel zeigt das Material in Abbildung 2. Es handelt sich um die Beschreibung einer zwar schon einige Jahre alten, nichtsdestoweniger aber aktuell für mein Thema aufschlussreichen Spielszene. 


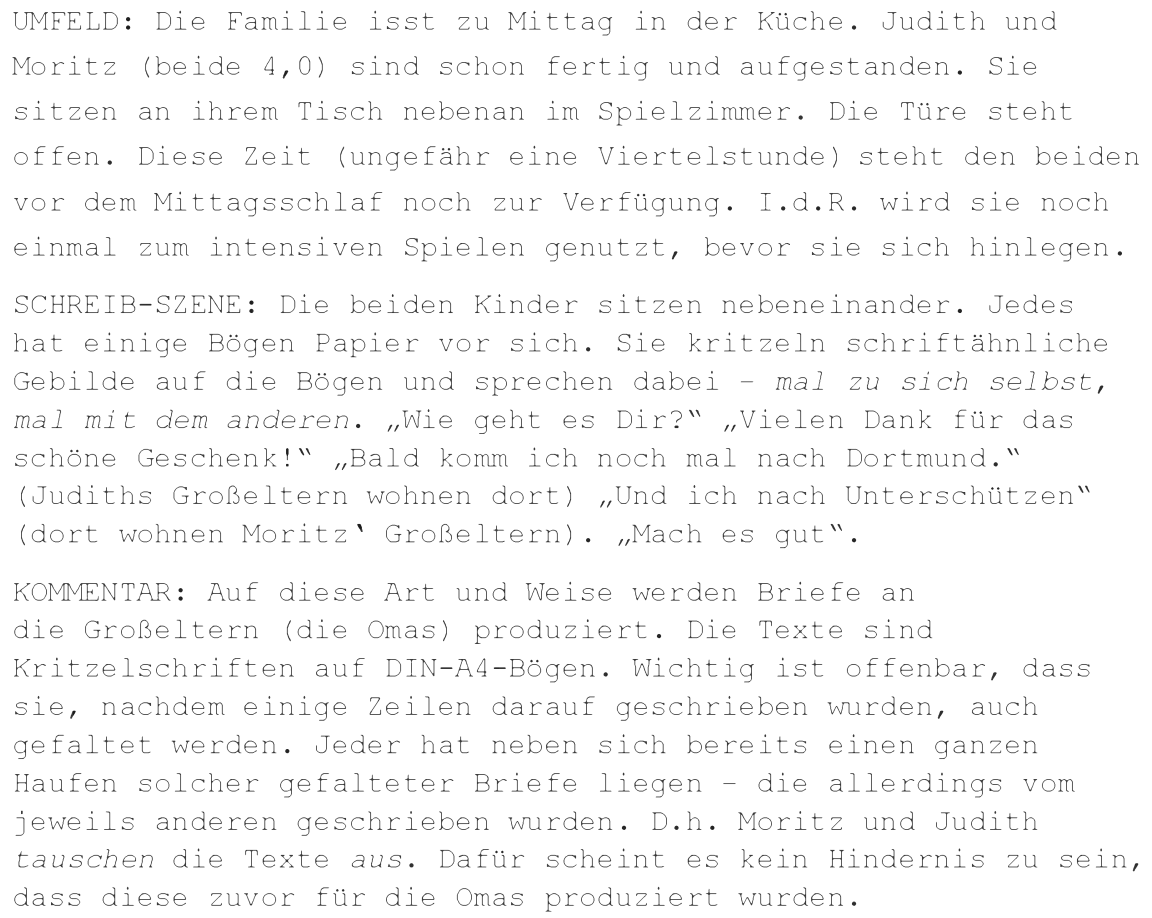

Abb. 2: Protoliterale Schreibszene (eigenes Protokoll: 25.9.1992)

Die Szene zeigt aus der Spracherwerbsforschung bekannte Merkmale des Spiels in diesem Alter, etwa die Idiosynkrasie von Parallelspiel (Sprechen zu sich selbst) und interaktivem Rollenspiel (Interaktion, Tausch der Briefe) (vgl. Andresen 2002). Thema des Spiels ist offenkundig das Briefeschreiben. Dies ist im soziologischen Sinne eine Praktik, das heißt ein intersubjektiv stabilisiertes Gefüge sozialer, am anderen orientierter Handlungsakte: Es gehört nicht nur der dazu, der schreibt, sondern auch der, der liest und wieder antwortet, sodass es zu einem Austausch kommt. Es gehören übliche Anlässe dazu und typische Themen und Konstellationen typischer Sender und Empfänger. Nach Reckwitz (2006, S. 38) besteht eine Praktik aus ,wiederholt hervorgebrachten Handlungsakten“. Sie ist

[...] von vorneherein sozial und kulturell, eine geregelte, typisierte, von Kriterien angeleitete Aktivität, die von verschiedensten Subjekten getragen wird. Wenn die Handlung per definitionem eine Intention impliziert, enthält die Praktik von vornherein einen Komplex von Wissen und Dispositionen, in dem sich kulturelle Codes ausdrücken (und die damit unter anderem auch typisierte Intentionen enthalten). (Reckwitz 2006, S. 38) 
Praktiken sind Einheiten des kulturellen Wissens: Das gilt auch hier. Die Kinder verdichten in ihrem Spiel die am Verhalten ablesbaren Komponenten der in natura räumlich, zeitlich und sozial distribuierten literalen Praktik: Sie schreiben, sie falten das Papier, sie übergeben Briefe und sie tauschen Briefe aus. Das heißt, sie haben offenbar ein Konzept, verfügen über einen „Komplex von Wissen und Dispositionen“ (Reckwitz) zu dieser literalen Praktik. Dies macht ihre Praktikenkompetenz aus. Die Kinder wissen, was man im konkreten Sinne tun muss, wenn man über Briefe kommuniziert. Und das ist das Erste, was sie lernen; das sozial sinnvolle Verhaltensmuster der Praktik ist nicht nur zeitlich, es ist auch pragmatisch prioritär gegenüber allen weiteren Komponenten einer literalen Kompetenz, der Kenntnis von Textsorten etwa, von Schriftwörtern oder Graphemen. Mit den Worten von Utz Maas: „Kulturelle Einheiten sind nicht einfach da - sie sind definiert durch den Umgang mit dem, was da ist" (Maas 2000, S. 15).

Diese Einsicht resultiert in einem dreistufigen Bild literaler Kompetenz, das ich unter die Stichworte: Kultur, Handlung und Struktur (vgl. Abb. 3 und Feilke 2014b) gebracht habe. Auf der primären Ebene geht es um die Antwort auf die Frage: „Wozu Schriftlichkeit?“ und die damit verbundenen Sinnressourcen für das Handeln. Sie werden angeeignet durch die Enkulturation in entsprechende Handlungsmuster, Einstellungen und Interessen. Auf der sekundären Ebene geht es um das hiervon abhängige Lese- und Schreibhandeln und die entsprechenden Prozesskompetenzen: Texte z.B. werden nicht per se geplant und überarbeitet, sondern abhängig von den auf der superstrukturellen Ebene der Praktiken etablierten Erwartungen, Interessen und Handlungsmotiven.

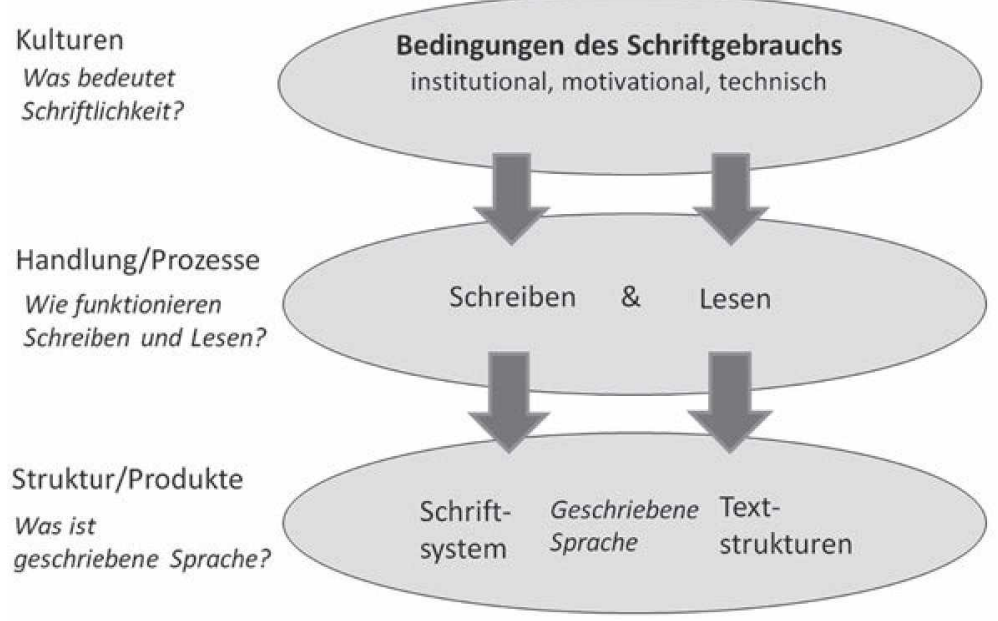

Abb. 3: Literale Kompetenz: Kultur - Handlung - Struktur (Feilke 2014b) 
Auf der dritten Ebene schließlich geht es um die resultierenden literal-sprachlichen Kompetenzen, die man etwa für das Briefeschreiben oder für das Schreiben eines wissenschaftlichen Textes braucht. Unter dem Begriff der geschriebenen Sprache sind dabei Aspekte des Schriftsystems ebenso wie des grammatischen und textlichen Duktus zu fassen. Zu Einzelheiten des Modells vgl. Feilke (2014b).

Die Spielszene illustriert allgemeine und spezifische Merkmale literaler Praktiken: In allgemeiner Perspektive ist die Domänengebundenheit und Situiertheit (Familienkommunikation), die Typisierung der Handelnden und ihrer Handlungen und der damit verbundenen Absichten innerhalb der Praktik im Beispiel ebenso erkennbar wie das Merkmal der Rekurrenz und Routiniertheit des Verhaltens. Es ist die sozial geteilte Form des Verhaltens, eine „skillful performance“ (Reckwitz 2003, S. 290), die die Praktik ausmacht, nicht die konkrete inhaltliche Füllung. Gerade das ist in diesem Schreibspiel, das ja ohne einen wirklich geschriebenen Text auskommt, sehr schön zu sehen.

Gegenüber den Differenzierungen der soziologischen Praktikentheorie erscheint die folgende Bestimmung literaler Praktiken von Barton/Hamilton (2000, S. 8) auf den ersten Blick relativ schlicht: „Literacy practices are the general cultural ways of utilising written language which people draw on in their lives." Aber es kommen gegenüber der Theorie sozialer Praktiken wesentliche Bestimmungselemente literaler Praktiken hinzu, die ebenfalls an der Spielszene gut aufzeigbar sind. Ich möchte sie unter drei Stichworte bringen: Abstraktheit literaler Praktiken, Textbezug als Definiens und die herausgehobene Rolle literaler Artefakte. Auch hierzu ein Zitat von Barton/Hamilton (ebd.): „Literacy is best understood as a set of social practices; these are observable in events, which are mediated by written texts.“

Abstraktheit: Das Zitat enthält mit der Unterscheidung von practice und event eine linguistisch vertraute Unterscheidung. „Literal events“ sind im Wortsinne je eventuelle, ereignishafte Gebrauchssituationen; verstanden werden sie dadurch, dass die Akteure sie kognitiv einem sinnvollen Verhaltensmuster, einer Praktik zuordnen können. Die Szene belegt m.E. die beachtliche Fähigkeit der Kinder zu einer abstrahierenden, durchaus konzeptionellen Fassung der Praktik des Briefeschreibens in toto. Es geht nicht primär um die in soziologischen Praktikentheorien stark betonte Inkorporierung von Bewegungsmustern. Vielmehr steuert das Musterwissen die intelligible und abstrahierte Ordnung des Briefeschreibens, das Praktikenspiel der Kinder. Das kann man schon daran sehen, dass der Austausch von Briefen empirisch nicht in der Weise stattfindet, wie ihn die Kinder spielen. Praktiken sind als Schematisierungen der Praxis abstrakte Größen. Es sind performative Types.

Textbezug: Die zweite wichtige Aussage des Zitats bezieht sich darauf, dass konkrete literal events wie dann auch die literal practices dadurch definiert sind, dass das Handeln der Akteure „mediated by written text“ ist. Hier ist das Handeln 
der Kinder freilich ,nur‘ im Modus des Spiels auf den „Text“ bezogen. Mit demselben Text kann auf unterschiedliche Weise umgegangen werden, er kann abgemalt und kopiert, gesungen, still gelesen oder analysiert werden. Aber er ist das Definiens für die Unterscheidung literaler von anderen Praktiken.

Literale Artefakte: „Wie geht es dir?“ „Vielen Dank für das schöne Geschenk!“ Dies sind hochgradig routinierte Sprechakte für Privatbriefe. Nicht zufällig bestimmen deshalb diese pragmatisch salienten und prominenten Textprozeduren (vgl. Feilke 2012b) die textbezogene Sprachproduktion der Kinder im Spiel. Für die Theorie literaler Praktiken sprechen Barton/Hamilton (2005) im Anschluss an Wengers Theorie der „Communities of Practice“ (1998) von Reifikationen und literalen Artefakten, ohne das Konzept extensional zu definieren:

\footnotetext{
In whatever way they are described, as cultural artefacts, mediating tools or stable mobiles, reifications are crucial for interactions across time and space, and often they are literacy artefacts. Reifications orchestrate and synchronise people's activities by stabilising meanings. (Barton/Hamilton 2005, S. 14)
}

Wo die Theorie literaler Praktiken an dieser Stelle linguistisch merkwürdig unbestimmt bleibt, kann man deutlicher werden: Es sind die pragmatisch signifikanten und deshalb rekurrenten Handlungszüge in Texten - ich spreche von Textroutinen oder Textprozeduren -, die als Artefakte reifiziert, das heißt in Form von Gliederungssignalen, Kollokationen und grammatischen Konstruktionen lexikalisiert und in dieser Weise verfügbar gehalten werden (vgl. Feilke 2012b, 2014a). Zusammen mit textsemantischen Konfigurationen bilden sie Textgenres, -sorten oder Gattungen mit einem erheblichen Kontextualisierungspotenzial für literale Praktiken.

Die Analyse der Spielszene kann verdeutlichen, dass die jüngere soziologische Praktikentheorie und das Konzept literaler Praktiken gut zu verbinden sind. Praktiken und die mit ihnen verbundenen Fähigkeiten der Individuen sind in der bis hierher vorgestellten Sicht emergente Folgen sozialer Ordnungsbildung im Schriftgebrauch, Phänomene der dritten Art - wie Keller (1990) das genannt hat - im Bereich der Schriftlichkeit. Erwerbs- und Aneignungsprozesse werden entsprechend in der soziologischen Praktikentheorie nach Bourdieu als Sozialisations- und Enkulturationsprozesse verstanden, deren wesentliches Kennzeichen eine „stille Pädagogik“ (Bourdieu 1987, S. 128) ist, deren Prinzip in einer „stummen Weitergabe“ (Schmidt 2008, 2012, S. 204 ff.) der Praktiken besteht:

Kernpunkt dieser alltäglichen und stillen Pädagogik ist eine Logik des Impliziten: Über stumme, gestische und körperliche Prozesse werden implizite Einstellungen, Werte, Kosmologien eben nicht explizit zum Gegenstand von Lern- oder Sozialisationsprozessen gemacht, sondern implizit übertragen. (Schmidt 2008, S. 124) 
Das gilt in gleicher Weise für die zur Praktik gehörenden Fertigkeiten. Es bleibt freilich zu klären, welches die notwendigen Bedingungen dafür sind, dass diese „Übertragung“ - etwa als ein Modelllernen im Sinne der Sozialpsychologie Banduras - auch aus der Sicht der Subjekte funktioniert. Noch spezifischer wäre nachzufragen: Ist literale Kompetenz tatsächlich zureichend nach der Idee einer aus den Umfeldern informeller Aneignungsprozesse erwachsenden „emergent literacy" zu verstehen?

Dagegen sprechen auf den ersten Blick zwei Aspekte, auf die im folgenden Kapitel näher eingegangen werden soll: Gerade der Erwerb literal-sprachlicher Kompetenzen ist schon historisch dadurch ausgezeichnet, dass er informeller Aneignung entzogen und der Schule als einer Institution formeller Bildung überantwortet wird. Außerdem wird gerade der Erwerb von Schriftlichkeit weithin als das beste Beispiel für eine notwendige Bewusstwerdung des Lernens und bewusster, grammatisch-expliziter Kontrolle der sprachlichen Verhaltensschemata thematisiert. Beide Punkte scheinen kaum zum praktikentheoretischen Konzept der „stummen Weitergabe“ zu passen.

\section{Schulische Literale Praktiken}

Warum werden Schreiben und Lesen in der Schule gelernt? In den Schriften unterschiedlicher Autoren zu literalen Praktiken wird immer wieder betont, dass von Literalität eigentlich nur im Plural zu sprechen sei, und dass abhängig von den Praktiken sich eine Vielzahl unterschiedlicher Literalitäten entwickele. Andererseits sind „literale Praktiken“ in allen Schriftkulturen durch eine enorme institutionale Asymmetrie und den Zug zu einer machtgestützten Homogenisierung gekennzeichnet. Brian Street, ein prominenter Vertreter der „New Literacy Studies"schreibt:

Literacy [...] is already part of a power relationship, and how people take hold of it, is contingent on social and cultural practices and not just on pedagogic and cognitive factors. (Street 2009, S. 337)

Die Schule ist ein Hauptakteur dieser machtbezogenen Homogenisierung literaler Praktiken. In diesem Sinn sind z.B. Abschreiben, Schönschreiben, Diktatschreiben zurecht als Beispiele für soziale Kontrollpraktiken kritisiert worden. Dies alleine kann m.E. allerdings kaum den engen Zusammenhang von „education und literacy“ erklären, zu dem Barton/Hamilton feststellen: „A study of literacy practices has its most immediate links with education.“ (Barton/Hamilton 2000, S. 14). Wo liegen die Ursachen dafür? 
Die spontane Entwicklung literaler Praktiken als Kommunikationsformen ist nicht der Prototyp des Literalitätserwerbs. Olson und Astington schreiben dazu in einer kritischen Entgegnung auf Tomasellos/Krugers/Ratners einflussreichen Aufsatz „Cultural learning“ von 1993: ,[...] it is not the ability to imitate that provides the basis for the accumulation of human cultures but the ability to teach" (Olson/Astington 1993, S. 532).

Der Literalitätserwerb findet angeleitet und durch die Einübung schulischer literaler Praktiken statt, die - eben als generalisierte Praktiken - zugleich auch kulturell mächtige Normen und weltanschaulich bestimmte Muster des Schriftgebrauchs etablieren. Gleichwohl stellt sich die Frage, wie sich unter einer praktikentheoretischen Perspektive der protoliterale Erwerb von Schriftlichkeit und der schulisch angeleitete Schriftspracherwerb zueinander verhalten.

Ganz offenkundig kann der Erwerb von Schriftlichkeit protoliteral durchaus im Sinne einer „stummen Weitergabe“ von Praktiken funktionieren, wie es im vorigen Kapitel am Beispiel der beiden spielenden Kinder vorgestellt worden ist. Wie aber verhält sich dazu der schulische Schriftspracherwerb? Ich möchte die Antwort in Form einer These geben, die ich dann an zwei Beispielen erläutern werde. Die These lautet:

Die Aneignung der sprachlichen Formen für eine raumzeitlich asynchron schriftliche Kommunikation - und darum geht es im Schriftspracherwerb verlangt einen hohen Grad formorientierter sprachlicher Aufmerksamkeit, Fokussierung und willkürlicher operationaler Kontrolle der Formelemente. Der Aufbau dieser Fähigkeiten profitiert von professionell unterstützender Anleitung und der methodisch strukturierten Aneignung einschlägiger metasprachlicher Praktiken.

Es werden deshalb (phonologisch, grammatisch und pragmatisch) genuin schulische literale Praktiken etabliert, die darauf bezogen sind, die entsprechende Aufmerksamkeit und Kontrollfähigkeit herzustellen. Die Praktiken lassen sich zusammenfassend als „Explizitheitspraktiken“ charakterisieren. Sie werden als konkrete Handlungsmuster und Maximen oder in Form von didaktischen Artefakten reifiziert und materialisiert.

\section{Erstes Beispiel: Praktiken phonologischer Bewusstwerdung}

Das Lesen und Schreiben einer Alphabetschrift verlangt die Zuordnung der Ergebnisse phonologischer Analysen zu Graphemen. Hierzu hat José Morais 1987 eine für das Thema klassische Untersuchung zur Entwicklung phonologischer Bewusstheit durchgeführt. Er weist nach, dass es zwar möglich, ohne einschlägige Instruktion aber unwahrscheinlich ist, dass Lerner die für die Segmentie- 
rung von Phonemen als rein formalen Strukturelementen notwendige Sprachaufmerksamkeit aufbringen.

Der entscheidende, den Prozess voranbringende Faktor liegt dabei den Ergebnissen Morais' zufolge nicht in den phonologischen Operationen wie Identifizieren, Segmentieren, Synthetisieren an sich. Vielmehr kommen diese erst vermittelt durch didaktische Praktiken des Zugriffs auf das Artefakt Alphabet „als Modell der Lautsprache“ (Günther 1995) entscheidend in Gang. Mit den Worten Morais' (1987, S. 130): „Acquiring full segmental awareness without literacy instruction is certainly not an impossible task, but it would require much more extensive training.“ „Segmental awareness“ ist hier ein Ergebnis der didaktischen Praktiken des Umgangs mit dem Alphabet. Die Wirkung des Artefakts beschreibt Stetter (1997) wie folgt:

Man projeziert für sich allein sinnlich nicht wahrnehmbare, weil kontinuierliche Elemente des gesprochenen Worts auf diskrete, für sich wahrnehmbare Zeichen. Erst in dieser Projektion gewinnt ,der` Laut seine Bestimmtheit. (Stetter 1997, S. 61)

Zur Veranschaulichung ein schönes Beispiel des ZEIT-Autors Ulrich Holbein, der sich in der Rubrik „Sprachlupe“ an sein erstes Schuljahr zurückerinnert:

\begin{abstract}
Mit sechs saß ich im Wohnzimmer vor meinem Täfelchen, sollte das Wort ESEL schreiben. Das E stand schon da. Doch das SEL ließ auf sich warten. Mutti sprach mir das SEL immer wieder vor, verriet aber nicht, wie man's schreibt. [...] Ich hielt das dauerhaft ausbleibende SEL für einen einzigen geheimnisvollen Buchstaben. Draußen wurde Roller gefahren. Ich klopfte alle mir bekannten Buchstaben der Reihe nach ab, aber SEL kam nicht. Einsam stand das E da; ich klagte, jammerte, weinte und kam nicht drauf. [...] (Ulrich Holbein, Vom Ursprung der Worte, ZEIT vom 17.5.1996)
\end{abstract}

Die phonologische Signatur von Phonemen als Referenten von Graphemzeichen ist in der phonetisch koartikulativen Einheit der Silbe nur schwer wahrnehmbar. Holbein hat durchaus schon eine Strategie: Er projiziert die wahrgenommene Einheit des Sprechens, die Silbe SEL, „der Reihe nach“ auf die diskreten Einheiten des Artefakts Alphabet, aber auch das will nicht gelingen. Wenn er allerdings die Projektionsrichtung umkehrte und ausgehend von den Buchstaben des geschriebenen Wortes fragte, hätte er eine Chance, SEL zu verstehen.

Die Praktiken dafür können im Unterricht methodisch in unterschiedlicher Weise ausgestaltet sein. Die geeigneten Praktiken weisen sich dadurch aus, dass sie das Ziel segmentaler phonologischer Bewusstheit stützen.

Freilich handelt es sich bei diesen in den Bereich formaler Bildung fallenden Handlungsmustern um etwas anderes als die emergenten Formen des Briefeschreibens. Aber es sind dies deshalb nicht weniger literale Praktiken als das 
Briefeschreiben. Konstitutiv sind die in hohem Maß routinisierten kommunikativen Verfahrensweisen und Handlungsmuster, die beteiligten Artefakte und deren Wahrnehmung durch die Akteure. Dabei geht es nicht primär um eine sprachlichkommunikative, sondern um eine metasprachliche Praktik, wie überhaupt literale Praktiken sich dadurch auszeichnen, dass sie als Praktiken mit starken metasprachlichen und metatextuellen Komponenten $\mathrm{zu}$ bestimmen sind.

Das ändert - wider Erwarten - gleichwohl nur wenig am praktikentheoretischen Prinzip einer ,impliziten Strukturvermittlung“ (Schmidt 2008, S. 124). Für Bourdieu ist es eine Tatsache, „daß die allgemeinen Prinzipien der Übertragung von Lernprozessen auch für die schulischen Lernprozesse gelten“ (Bourdieu 1974, S. 188). Sie werden in einen institutionalen Rahmen verlagert und gewissermaßen kognitiv stärker enggeführt. Kinder werden als Schüler zu Mitgliedern einer Institution, die als solche bestimmte Gegenstände und darauf bezogene Verhaltensmuster (etwa des analysierenden Umgangs mit Schriftwörtern, bei Bourdieu sind es Kunstwerke) verbindlich macht. Methoden, verstanden als soziale Praktiken schulischer Unterrichtung, formen in der Ausrichtung auf Artefakte (z.B. das Alphabet, ausgewählte Beispielwörter oder Beispielsätze) das konkrete Handeln und erzeugen damit Sprachaufmerksamkeit und die gewünschten kognitivsprachlichen Dispositionen und Einstellungen. Nun könnte man entgegnen, der Unterricht sei dann doch gerade keine „stumme Weitergabe“, sondern diene der Explizierung des zu Vermittelnden. Das ist einerseits unzweifelhaft der Anspruch. Andererseits ist die Methodizität des Unterrichts in erster Linie ein Verfahren, einen Habitus zu reproduzieren, das heißt, eine gewohnheitsmäßige Form des Umgangs mit bestimmten Problemen und Gegenständen. Erfolgreiche Schüler lernen zunächst, welches Verhalten von Ihnen erwartet wird. Wenn der Unterricht erfolgreich ist, entdecken sie innerhalb der weitergegebenen Verhaltensmuster, worauf es ankommt.

\section{Zweites Beispiel: Praktiken des Texteschreibens}

Auch im folgenden Beispiel wird exemplarisch die Rolle literaler Artefakte deutlich - in diesem Fall einer didaktischen Gattung, der Inhaltsangabe. Das Transkript entstammt einer Gießener Staatsexamensarbeit, in der unterschiedliche Formen kooperativen Schreibens im Blick auf ihre Lernwirksamkeit verglichen wurden. Es geht um eine Schreibkonferenz im 9. Schuljahr eines Gymnasiums. Drei Schüler, Denise, Yasmin und Kevin, - es handelt sich nicht um die echten Namen - haben Inhaltsangaben zu einer Kurzgeschichte (hier „Brudermord im Altwasser“ von Georg Britting 1929) geschrieben, die nun untereinander besprochen werden sollen. Zuerst fordern die Mädchen Kevin auf, seinen Text vorzulesen: 


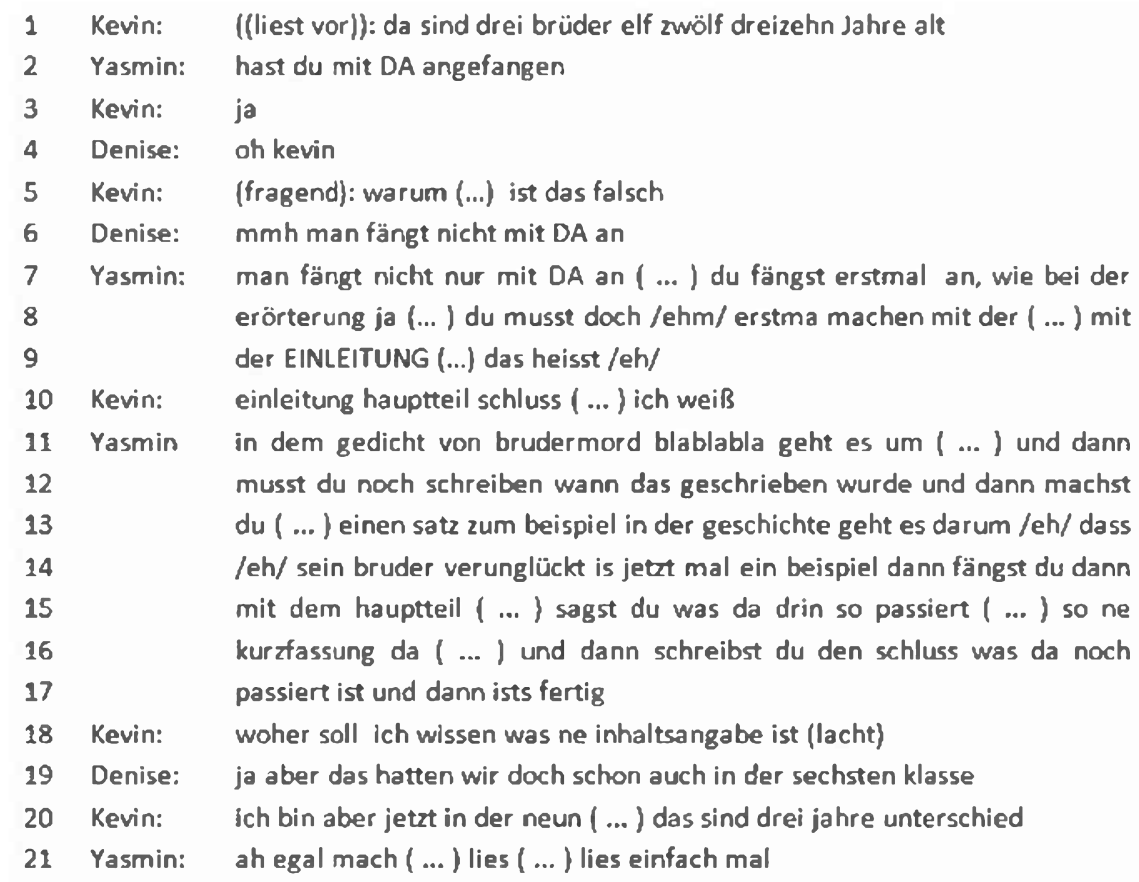

Abb. 4: Gesprächsausschnitt Kevin, Yasmin, Denise (Lüling 2014, S. 59; Transkript der Autorin)

Zwei Beobachtungen nur möchte ich an diesem Beispiel hervorheben. Die Schreibkonferenz funktioniert als Teil eines Zusammenhangs unterrichtlicher Schreibpraktiken, die aufeinander bezogen sind: Schreiben, Schreibkonferenz mit Diskussion des Textes und schriftlicher Kommentierung und Überarbeitung. Dieser methodisch konzipierte Zusammenhang ist ähnlich wie die methodischen Teilschritte der Phonemidentifizierung und -manipulation als didaktische Praktik zu analysieren. Kevins Text hat am Ende dieses Prozesses deutlich gewonnen, worauf ich hier nicht eingehen kann. Integriert und gesteuert wird dieser Zusammenhang im Dialog der Schüler wiederum durch ein Artefakt, in diesem Fall mit Bezug auf die Anforderungen der didaktischen Gattung „Inhaltsangabe“: „man fängt nicht mit DA an“ (6).

Was Denise hier gattungsbezogen als Maxime fomuliert, verweist wiederum auf das übergreifende Schema der nunmehr auf der Textebene zum Zug kommenden Explizitheitspraktik, vor allem erkennbar in den Äußerungen 2, 6, 7-9 und 11-17. Das von Kevin unmittelbar auf die Geschichte bezogene deiktische $d a$ setzt für den nicht mit dem zu referierenden Text vertrauten Leser zunächst eine einleitende Herausstellung und Explizierung des Gegenstandes und Themas der Inhaltsangabe selbst voraus. Sie wird von Yasmin in Äußerung 7-9 - unter Bezug 
auf die gleichen Muster einer anderen didaktischen Gattung, der Erörterung eingeklagt. In Äußerung 11-17 wird positiv erkennbar, welche Komponenten in der Wahrnehmung der Schüler mit Notwendigkeit zu explizieren sind. Hier stellt Yasmin in einem imitierenden Modus das Grundgerüst eines Inhaltsreferats vor. Die Gattung als Artefakt wird repräsentiert durch die hier zitierten Textprozeduren, ganz ähnlich, wie dies auch in der protoliteralen Briefszene erkennbar war.

Es ist produktiv, das Praktikenkonzept auch auf Praktiken in Institutionen, hier auf Praktiken formaler Bildung zu beziehen. Dies kann insbesondere auch im Blick auf die Rekonstruktion der Akteursperspektiven - namentlich der Schüler - ein Gewinn sein. Der vertraute didaktische Methodenbegriff artikuliert im besten Fall die fachlich besonnte Seite des Mondes unterrichtlicher Praktiken. Ein Reden von Praktiken statt von Methoden kann nicht zuletzt auch die Chance eröffnen, einen Blick auf die unbeleuchtete Seite des Unterrichtsgeschehens zu gewinnen. Für Schüler sind Inhalt und Ziel des Unterrichts naturgemäß zunächst dunkel und intransparent; was sie - als Schüler - zuerst verstehen müssen und auch verstehen, sind die Praktiken der Problembearbeitung im Unterricht und die Anforderungen an ihren „Schülerjob“ in diesen Praktiken - wie Breidenstein (2006) das nennt. Interessant und für den Erwerb relevant ist dann die empirische Frage, wie sich die Wahrnehmung der schulischen literalen Praktiken bei Lehrern und Schülern unterscheidet.

Die phonologische Explizierung und die Explizierung des Referenztextes in einem Textreferat sind nur Beispiele. Literale Explizierungspraktiken kommen in vielerlei kommunikativer Form und Gestalt - medial mündlich wie schriftlich vor. Sie prägen die Schulsprache (Feilke 2012a). Mary Schleppegrell schreibt in ihrem Buch „Schooled Language“ dazu:

Even from the earliest school tasks, teachers have certain expectations of students for how they should use language. These expectations are typically not articulated in terms of grammatical or discourse structure, but instead in terms of admonitions to ,be explicit', be ,more detached', or be ,better organized'. (Schleppegrell 2010, S. 25)

Explizitheitspraktiken sind bildungssprachliche Praktiken im Sinn des folgenden hilfreichen Definitionsvorschlags von Morek/Heller (2012):

Unter bildungssprachlichen Praktiken verstehen wir somit die (vorzugsweise in Bildungsinstitutionen) situierten mündlichen wie schriftlichen sprachlich-kommunikativen Verfahren der Wissenskonstruktion und -vermittlung, die stets auch epistemische Kraft entfalten (können) und zugleich bestimmte bildungsaffine Identitäten indizieren. Diese Verfahren erhalten den Status sozial etablierter Praktiken erst und gerade dadurch, dass sie von erfahrenen Agenten der Institution normativ sowohl implizit als auch explizit eingesetzt und auch aktualisiert werden. (Morek/Heller 2012, S. 92) 
Explizitheitspraktiken im Sinne bildungssprachlicher Praktiken wiederholen sich auf der grammatischen Ebene des Sprechens und der Redeorganisation ebenso wie beim Schreiben von Texten. Wichtig ist, dass es hier um mehr als ein Normwissen geht: Eine bloße Explizitheitsnorm bliebe sozial ohne Substanz, wenn es die rekurrenten kommunikativen Muster ihrer Herstellung und Reproduktion nicht gäbe. Hier wie dort geht es darum, durch die Praktik und die ihr korrespondierenden Handlungen der Lerner die tragenden Komponenten der neu zu erwerbenden Kompetenz herauszustellen und willkürlich kontrollierbar zu machen.

\section{Wissenschaftliche literale Praktiken}

Das Beispiel zur Inhaltsangabe leitet über zum letzten Abschnitt dieses Beitrags. Inhaltsangaben sind die Schulform des Textreferats, und was dieser Schulform in den meisten Fällen fehlt, ist wiederum die didaktische Situierung in einer sozialen Praktik des Referierens. Das dürfte zumindest ein Grund dafür sein, dass sie unter Schülern zu den deutlich weniger beliebten Schreibformen zählt (Fix 2000, S. $116 \mathrm{ff}$.). Das ist bedauerlich, und es ist unter wissenschaftspropädeutischen Gesichtspunkten ein großer Mangel.

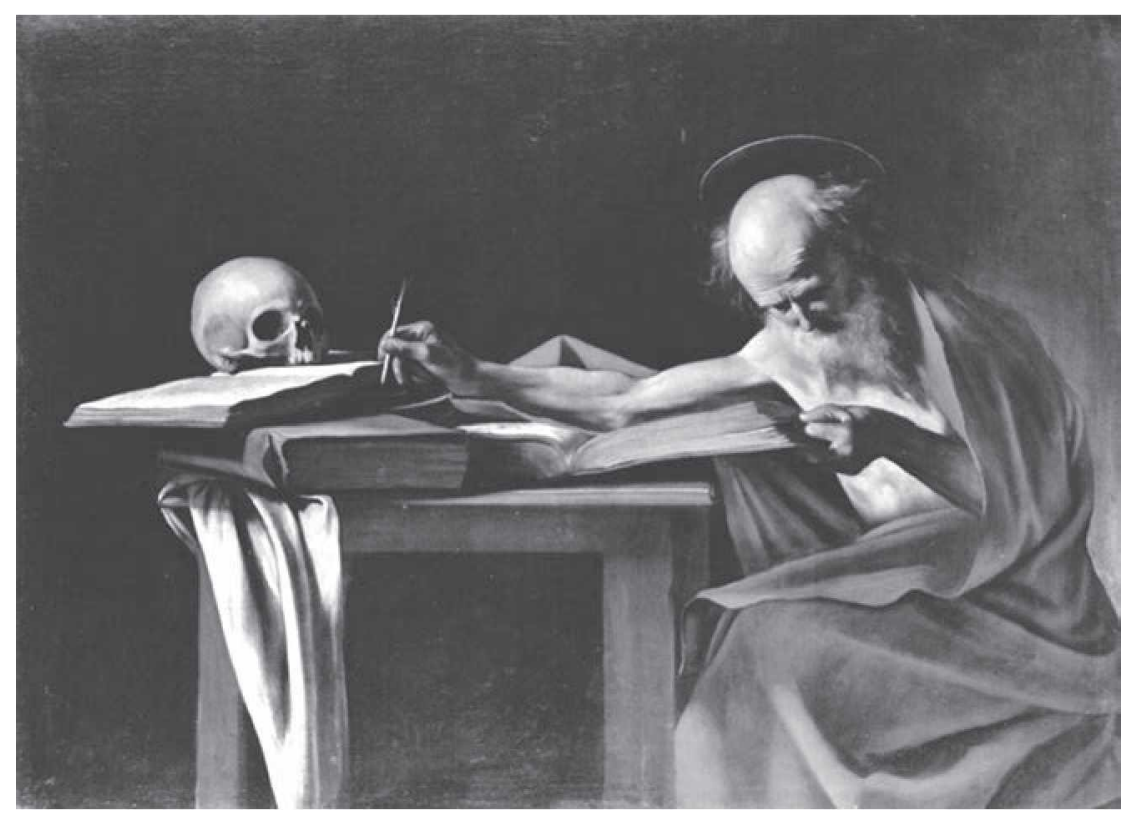

Abb. 5: Caravaggio: „Der heilige Hieronymus, schreibend“ (um 1606) 
Wissenschaftlich kommt es darauf an zu verstehen, dass das Schreiben ein textverarbeitendes Schreiben, ein „writing from sources“ (Jakobs 1999) ist und dass das Lesen die Inhalte fremder Texte mit Blick auf deren Verstoffwechslung im eigenen Schreiben verarbeitet: ,reading-to-write“ hat Linda Flower das genannt (Flower/Long/Higgins 2000; Feilke/Lehnen 2011). Ein solches Bild des Schreibens zeigt das für mich eindrucksvolle Gemälde Caravaggios aus dem Jahr 1606 in Abbildung 5, das den Titel trägt: „Der Heilige Hieronymus, schreibend“.

Wissenschaftliches Schreiben ist als literale Praktik dadurch bestimmt, dass eine Fragestellung unter Rückgriff auf den Forschungsstand und unter Berücksichtigung und Integration multipler Textquellen in den Schreibprozess bearbeitet wird. Der textliche Modus dieser intertextuellen Praktik, die ,aus vielem eines schafft" - um eine Anspielung aus der Einleitung der unrühmlich bekannt gewordenen Dissertation des Ministers Guttenberg zu bemühen - ist das Zitieren. Texte anderer werden für die eigene Problembearbeitung und Argumentation genutzt. Erst das macht den wissenschaftlichen Autor aus (vgl. Feilke 2010). Sehr gut fasst diesen Zusammenhang das folgende Zitat von Bonaventura, das Ivan Illichs (1991) Buch „Im Weinberg des Textes“ entnommen ist.

Es gibt vier Arten, ein Buch zu machen. Man kann Fremdes schreiben, ohne etwas hinzuzufügen oder zu verändern, dann ist man ein Schreiber (scriptor). Man kann Fremdes schreiben und etwas hinzufügen, das nicht von einem selbst kommt, dann ist man ein Kompilator (compilator). Man kann auch schreiben, was von anderen und von einem selbst kommt, aber doch hauptsächlich das eines anderen, dem man das Eigene zur Erklärung beifügt, und dann ist man ein Kommentator (commentator), aber nicht ein Autor. Man kann auch Eigenes und Fremdes schreiben, aber das Eigene als Hauptsache und das Fremde zur Bekräftigung beifügen, und dann muß man als Autor (auctor) bezeichnet werden. (Bonaventura (14. Jahrhundert), zit. nach Illich (1991, S. 112 f., ohne Beleg)

Bei Licht betrachtet unterscheidet Bonaventura hier vier verschiedene Praktiken des Schreibens mit Bezug auf fremde Texte. Sie können eine produktive Heuristik für die Differenzierung von Schreibkulturen bilden. Der letzte Punkt, die Autorschaft und damit epistemische Originalität, ist eines der zentralen Kriterien wissenschaftlichen Schreibens. Das Zitieren ist der Modus einer argumentativ-dialogischen Auseinandersetzung mit den Positionen anderer im Fachdiskurs, wie dies Kretzenbacher im folgenden Zitat sehr klar formuliert.

Der Dialog wird mit dem intertextuellen Mittel des Zitats als Berufung auf unterstützende und als Auseinandersetzung mit konkurrierenden Theorien sowie intratextuell als argumentative Antizipation möglicher Einwände oder Verständnisschwierigkeiten der Leserschaft ausgetragen. (Kretzenbacher 1997, S. 137) 
Konrad Ehlich (1993) hat diesen Modus des Schreibens und den entsprechenden Aufbau wissenschaftlicher Texte ,eristisch“ genannt, das heißt soviel wie: auf den epistemischen Streit und die epistemische Kontroverse hin angelegt. Hierauf bezieht sich ein Gießener VW-Projekt (2012-2016) zum Erwerb der eristischen Literalität in der Wissenschaftssprache Deutsch bei germanistischen Masterstudierenden mit Deutsch als L1 und L2. ${ }^{3}$

Uns interessiert, was studentische Schreiberinnen und Schreiber sprachlich zu Autor/innen ihrer Texte macht: Wie schaffen sie es, unterschiedliche wissenschaftliche Äußerungen zu einem kontroversen Thema so im eigenen Text zu verarbeiten, dass es nicht zu einer bloß aggregativ-reproduzierenden Wiedergabe kommt, sondern eine integrativ-synthetisierende, diskursive Themenbehandlung erreicht wird? Was zeigen hierzu makro- und mikrostrukturell die entstehenden Texte als Produkte? Und was kennzeichnet in dieser Hinsicht die Lernprozesse Studierender? Das Projekt kann hier nicht im Detail vorgestellt werden; an einem Beispiel soll aber abschließend gezeigt werden, wie empirische Beobachtungen auf Fragen des Erwerbs der literalen Praktik wissenschaftlichen Schreibens im oben erläuterten Sinn bezogen werden können.

Ein aufschlussreicher Beobachtungspunkt im Bereich der sprachlichen Mikrostruktur eristischer Schreibpraktiken sind die syntaktischen Formate der Integration von Zitaten in den eigenen Text. Routiniertheit der literalen Praktik wissenschaftlichen Zitierens zeigt sich darin, dass ein Schreiber in der Lage ist, eine Fremdäußerung zugleich wiederzugeben, sie zu bewerten und - streitend - auf aktuelle kontroverse Gesichtspunkte der Fachdiskussion zu beziehen. Hochgradig indikativ in dieser Hinsicht ist der in Abbildung 6 dargestellte Typ grammatischer Konstruktionen.

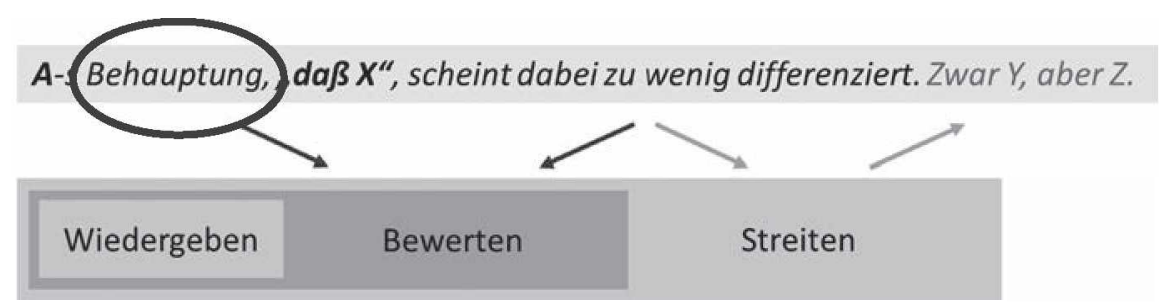

Abb. 6: Zitatrahmen als Scharnierstellen (Steinseifer i.Vorb.)

3 Eristische Literalität. Antragsteller: Helmuth Feilke und Katrin Lehnen, Verantwortliche Mitarbeiter: Martin Steinseifer, Lisa Schüler, Volker Emmrich. Siehe www.uni-giessen.de/fbz/zmi/ projekte/eristischeliteralitaet. 
Martin Steinseifer, der diese Konstruktionen im Projekt genauer untersucht, nennt sie Zitatrahmen. Sie bilden Scharnierstellen für die Verbindung von Wiedergabe und Bewertung des Zitats im Blick auf den eigenen Textzusammenhang. Wiedergeben und Bewerten sind dabei beide schon bezogen auf das Streiten. Die Bewertung bildet die Grundlage für die weiterführende Diskussion. Wir sehen Konstruktionen eines solchen Typs in den Texten der Studierenden als Indikatoren eristischer Kompetenz. Auf einen letzten Punkt möchte ich an dem Beispiel aufmerksam machen. Nomina wie Behauptung, These, Auffassung in der grammatischen Konstruktion, nennen wir „Zitatnomen“.

\begin{tabular}{rrrrrr}
\multicolumn{7}{c}{ ZN Anzahl } & & & \\
\hline 1 & These & 15 & 11 & Befund & 4 \\
2 & Annahme & 13 & 12 & Einsicht & 4 \\
\hline 3 & Erkenntnis & 13 & 13 & Gedanke & 4 \\
4 & Eindruck & 9 & 14 & Voraussetzung & 4 \\
5 & Ergebnis & 8 & 15 & Hinweis & 3 \\
\hline 6 & Auffassung & 7 & 16 & Hoffnung & 3 \\
\hline 7 & Feststellung & 7 & 17 & Hypothese & 3 \\
8 & Überzeugung & 6 & 18 & Meinung & 3 \\
9 & Schluss & 5 & 19 & Überlegung & 3 \\
10 & Vorstellung & 5 & 20 & Vermutung & 3
\end{tabular}

Abb. 7: Zitatnomen (Steinseifer i.Vorb.), Basis: Expertentexte-Korpus Steinhoff (2007)

Über Zitatnomen zeigt der Schreiber, wie er das anschließende Zitat epistemisch und auch rhetorisch verstanden wissen möchte, als Hypothese, als These oder z.B. als Meinung. Zugleich zeigt dies aber auch, wie der Schreiber selbst den Fachdiskurs auffasst, z.B. als Gemengelage von bloßen Meinungen, wo der eine dies und der andere eben das meint, oder als eine argumentative Auseinandersetzung über sachhaltige Thesen, deren Haltbarkeit zu prüfen ist. Wir haben deshalb die Zitatnomen genauer untersucht (vgl. Abb. 7). Aus dem Expertentextekorpus der Arbeit von Steinhoff (2007) „Wissenschaftliche Textkompetenz“ haben wir die Zitatnomen-Types extrahiert. Auch hier spiegelt sich der angesprochene Unterschied zwischen eher thesenbetonten und eher meinungsbetonten Zitatnomen. $\mathrm{Zu}$ den ersteren können etwa These und Annahme gezählt werden, zu meinungsbetonten zählen wir z.B. Auffassung oder Meinung. Im Expertenkorpus führen die thesenbetonten Nomina These und Annahme die Liste an. 
In einer Pilotuntersuchung haben wir an einem kleinen Teilkorpus unserer Erhebung die Zitatnomenvorkommen bei von Ratern als gut und schwach eingeschätzten L1-Studierendentexten und L2-Studierendentexten ausgewertet. Bei aller Vorsicht, die gegenüber der Auswertung hier noch angebracht ist, zeigt sich, dass bei den Studierenden insgesamt die thesenbetonten Zitatnomen, die geeignet wären, den studentischen Verfasser als Autor im kontroversen Fachdiskurs auszuweisen, am wenigsten zum Einsatz kommen (zwischen 11\% und 24\% der Vorkommen je Gruppe). Es dominieren in allen Gruppen die meinungsbetonten Zitatnomina (zwischen 34\% und 64\% der Vorkommen je Gruppe). Dies kann bei Licht betrachtet, kaum verwundern, und es verwundert auch nicht, dass die bisher in die Untersuchung einbezogenen L2-Studierenden in dieser Hinsicht noch einmal stärker zurückhaltend sind als ihre muttersprachlich deutschen Mitstudierenden.

Interessant ist noch ein weiteres Resultat der bisherigen Auswertung. Wir haben verschiedene Konstruktionstypen für das Vorkommen von Zitatnomina unterschieden (vgl. Abb. 8).

Von den verschiedenen syntaktischen Kontexten, in denen Zitatnomen vorkommen können, ist der eingangs vorgestellte attributive Typ, also z.B. „Die Meinung, dass X, erscheint fragwürdig“ in unserem Korpus der deutlich am häufigsten gebrauchte Typ, und zwar mit einem Anteil von über 40\% bei allen unterschiedenen Studierendengruppen gleichermaßen. Daraus kann man folgern: Auch wenn der Gebrauch von Zitatrahmenkonstruktionen pragmatisch und semantisch noch große Unterschiede zu den Experten aufweist, die syntaktisch routinehaften Grundlagen für eine sukzessive Fortentwicklung des Handlungsschemas sind gelegt.

\begin{tabular}{|l|l|}
\hline $\begin{array}{l}\text { Isolierter Typ: } \\
\text { keine konstruktional angebundene Wiedergabe }\end{array}$ & X vertritt eine andere Meinung \\
\hline $\begin{array}{l}\text { Deiktischer Typ: } \\
\text { Wiedergabe geht dem Zitatnomen textuell } \\
\text { voraus }\end{array}$ & ZITAT. Diese These... \\
\hline $\begin{array}{l}\text { Attributiver Typ: } \\
\text { Wiedergabe ist über das Zitatnomen syntaktisch } \\
\text { integriert }\end{array}$ & $\begin{array}{l}\text { Die These, dass... } \\
\text { Die Behauptung, es gebe... }\end{array}$ \\
\hline $\begin{array}{l}\text { Parenthetischer Typ: } \\
\text { Zitatnomen ist eingebettet in die Wiedergabe }\end{array}$ & $\begin{array}{l}\text {..., so die These von } X, \ldots \\
\text { naciner Ansicht,... }\end{array}$ \\
\hline
\end{tabular}

Abb. 8: Konstruktionstypen für Verwendung von Zitatnomina (Steinseifer i.Vorb.) 
Es geht bei diesem Schlaglicht auf die Mikrostruktur der Intertextualität im wissenschaftlichen Schreiben nicht nur um den Erwerb sprachlicher Ausdrucksmöglichkeiten. Gleichermaßen geht es um die Aneignung einer literalen Praktik, die Wissenschaft als offenen und kontroversen Diskurs stützt. Literale Praktiken - darauf weist Street hin - haben schon für sich betrachtet stets auch epistemologische Implikationen. Er schreibt: „Literacy is a social practice, [...] and it is always embedded in socially constructed epistemological principles [...]“" (Street 2001, S. 8). Dieser Grundgedanke kann mit Ken Hyland bezogen auf die literalen Praktiken wissenschaftlichen Schreibens noch weitergehend spezifiziert werden:

[...] our routine and unreflective writing practices are deeply embedded in the epistemological and social convictions of our disciplines. (Hyland 1999, S. 363)

Es geht dementsprechend bei einem Konstruktionstyp wie der Zitatrahmenkonstruktion nicht nur um eine sprachliche Form. Die mikrostrukturelle Form ist motiviert durch konventionelle koordinierte Handlungszüge eristischen Argumentierens, denen auf der makrostrukturellen Ebene ein gattungsbildendes Potenzial zukommt. Und diese Handlungsstruktur wiederum hat ihren institutionellen Ort in einem Wissenschaftsverständnis und einer Wissenschaftskultur, die in den Praktiken der Kontroverse eine Quelle intersubjektiv gültiger Erkenntnis sehen.

\section{Schlusskommentar und Ausblick}

Praktikentheoretische Konzepte spielen in der Forschung zu Literalität und Schriftlichkeit eine wichtige Rolle, weil die sozial hervorgebrachten Gebrauchsformen der Schrift entscheidend sind für den Erwerb konzeptioneller Literalität. Im Unterschied zum primären Spracherwerb ist der Literalitätserwerb in hohem Maß institutional bestimmt. Deshalb ist es produktiv, literale Praktiken als institutional geformte Praktiken zu verstehen und umgekehrt Methoden des Unterrichts aus praktikentheoretischer Perspektive zu analysieren. Die in diesem Beitrag untersuchten Beispiele belegen die besondere Bedeutung von - oft didaktisch überformten - Artefakten (z.B. Alphabet als Modell der Lautsprache, didaktische Gattungen) für den Erwerb. Auch Textprozeduren sind in diesem Sinn für didaktische Zwecke modellierbare Artefakte. Der vorschulische Literalitätserwerb ist nach Bourdieus Konzept einer „stummen Weitergabe“ von Praktiken durch eine imitierende Übernahme von Verhaltensmustern und einen impliziten Strukturaufbau geprägt. Die spezifisch schulischen Lernkontexte nutzen diesen sozialen Lernmechanismus: Weil die Kognitionen der Lernenden den Lehrenden kaum zugänglich sind, werden Aufmerksamkeit und die Zuwendung zu den Lern- 
gegenständen - Schrift, geschriebene Sprache, Texte - über Artefakte und insbesondere über Methoden als im Unterricht sozial koordinierte Verhaltensmuster hergestellt. Sie sollen es ermöglichen, das Lernen am resultierenden Verhalten zu kontrollieren. Das ist freilich nicht ohne Risiko: Schüler lernen auch, ihren Aufwand $\mathrm{zu}$ minimieren und lediglich erwarteten Verhaltensmustern $\mathrm{zu}$ entsprechen. Eine praktikentheoretische Perspektive kann den Blick für diese Ambivalenz von Methoden öffnen. Hier liegt ein für Forschungen zum schulischen Spracherwerb wichtiges empirisches Forschungsfeld.

Es ist kein Zufall, dass ausgerechnet die Domäne der Wissenschaft ein besonders prominentes Anwendungsfeld praxistheoretischer Ansätze ist. Der Idee vom genialen Entdecker- und Forscherindividuum korrespondiert das soziale Denkkollektiv im Sinne Ludwik Flecks (1980), und dieses Kollektiv ist vor allem ein Kollektiv bestimmter sozialer Praktiken in der Hervorbringung von Erkenntnis. Auch dieser Argumentationsgang landet beim Begriff der Methode als sozialer Praktik: Da die ultima ratio von Wissenschaft (im Unterschied zu Weisheit und Eingebung) nicht in Erkenntnis an sich besteht, sondern in deren Nachvollziehbarkeit, kommt den sozialen Formen des Nachvollzugs und deren sprachlicher Gestalt eine entscheidende Rolle zu. Auch hier ist es die äußere Form sozialer Praktiken, insbesondere der sprachlichen Darstellung von Wissenschaft, die ein wichtiges Kriterium für die Überprüfung wissenschaftlicher Kompetenz bildet. Und auch hier liegt das aktuell vieldiskutierte Risiko darin, dass an die Stelle der Darstellung von Wissenschaft Wissenschaftsdarsteller treten können, die in erster Linie der Form der sozialen Praktik zu entsprechen vermögen. Gleichwohl ist es eine empirisch zentrale Forschungsaufgabe, $\mathrm{zu}$ untersuchen, worin genau sprachlich die typisierten Handlungsmuster bestehen. Die soziale Praktik z.B. wissenschaftlicher Kontroversität schlägt sich in einem breiten Spektrum sprachlicher Formen - etwa den hier vorgestellten Zitatrahmenkonstruktionen - nieder. Die Forschung zur Aneignung der entsprechenden Formen kann einen Beitrag zur Förderung der für wissenschaftliches Schreiben einschlägigen Kompetenzen leisten.

Literale Praktiken sind nach meiner Einschätzung ein produktives Anwendungsfeld für praktikentheoretische Konzepte in der Linguistik. Ganz sicher kann man, was z.B. in der Aneignung der Wissenschaftssprache Deutsch beim Schreiben beobachtbar ist, auch ganz ohne praktikentheoretische Konzepte beschreiben und untersuchen, z.B. Anforderungen der Institution, soziolinguistische Aspekte von Spracheinstellungen, sprachbezogene Erwartungen der Studierenden, Sprechhandlungstaxonomien in der Domäne Wissenschaft, Textsortenkonzepte, komplexe Handlungen in Texten, Grammatik und Lexik des Definierens, des Zitierens usw. Und in der Tat gibt es auch Arbeiten zu allen diesen und vielen weiteren Bereichen. Der praktikentheoretische Zugang bietet zum einen eine Mög- 
lichkeit, nach den Zusammenhängen zwischen diesen unterschiedlichen Analysebereichen zu fragen. Zum anderen ist der Zugang der Theorie sozialer Praktiken genuin soziologisch und stellt damit - zumindest für einen Teil der Linguistik zunächst eher „fremde“ Kategorien zur Verfügung: Macht und Autorität, Habitus, Rollenkonstellationen und Beteiligungsstrukturen, Körperlichkeit usw. Darin sehe ich eine Chance, linguistisch möglicherweise vernachlässigte, aber relevante Größen in die Analyse mit einzubeziehen, aber auch eine Option, aus der Perspektive der Linguistik fachlich eigenständige Argumente zur Konzept- und Theorieentwicklung beizutragen.

\section{Literatur}

Andresen, Helga (2002): Interaktion, Sprache und Spiel. Zur Funktion des Rollenspiels für die Sprachentwicklung im Vorschulalter. Tübingen.

Barton, David/Hamilton, Mary (2000): Literacy practices. In: Barton, David/Hamilton, Mary/ Ivanič, Roz (Hg.): Situated literacies: Reading and writing in context. London, S. 7-15.

Barton, David/Hamilton, Mary (2005): Literacy, reification and the dynamics of social interaction. In: Barton, David/Tusting, Karin (Hg.): Beyond communities of practice: Language power and social context. (= Learning in Doing). Cambridge, S. 14-35.

Berger, Peter L./Luckmann, Thomas (1980): Die gesellschaftliche Konstruktion der Wirklichkeit. Eine Theorie der Wissenssoziologie. Frankfurt a.M. [Dt. Erstaufl. Frankfurt a.M. 1969.]

Bourdieu, Pierre (1974): Zur Soziologie der symbolischen Formen. (= Suhrkamp-Taschenbücher Wissenschaft 107). Frankfurt a.M.

Bourdieu, Pierre (1987): Sozialer Sinn. Kritik der theoretischen Vernunft. Frankfurt a.M.

Breidenstein, Georg (2006): Teilnahme am Unterricht. Ethnographische Studien zum Schülerjob. (= Studien zur Schul- und Bildungsforschung 24). Wiesbaden.

Brockmeier, Jens (1997): Literales Bewußtsein. Schriftlichkeit und das Verhältnis von Sprache und Kultur. München.

Bühler, Karl (1982): Sprachtheorie. Die Darstellungsfunktion der Sprache. Stuttgart/New York. [Originalausg. Jena 1934.]

Coulmas, Florian (1981): Über Schrift. (= Suhrkamp-Taschenbuch Wissenschaft 378). Frankfurt a.M.

Ehlich, Konrad (1993): Deutsch als fremde Wissenschaftssprache. (= Jahrbuch Deutsch als Fremdsprache 19). München, S. 13-42.

Feilke, Helmuth (2003): Entwicklung schriftlich-konzeptualer Fähigkeiten. In: Bredel, Ursula et al. (Hg.): Didaktik der deutschen Sprache. Ein Handbuch. Paderborn, S. 178-192.

Feilke, Helmuth (2010): Schriftliches Argumentieren zwischen Nähe und Distanz: Am Beispiel wissenschaftlichen Schreibens. In: Ágel, Vilmos/Hennig, Mathilde (Hg.): Nähe und Distanz im Kontext variationslinguistischer Forschung. (= Linguistik - Impulse \& Tendenzen 35). Berlin/NewYork, S. 209-231.

Feilke, Helmuth (2012a): Schulsprache - Wie Schule Sprache macht. In: Günthner, Susanne et al. (Hg.): Kommunikation und Öffentlichkeit. Sprachwissenschaftliche Potenziale zwischen Empirie und Norm. (= Reihe Germanistische Linguistik 296). Berlin, S. 149-175. 
Feilke, Helmuth (2012b): Was sind Textroutinen? Zur Theorie und Methodik des Forschungsfeldes. In: Feilke, Helmuth/Lehnen, Katrin (Hg.): Schreib- und Textroutinen. Theorie, Erwerb und didaktisch-mediale Modellierung. (= Forum angewandte Linguistik 52). Frankfurt a.M. u.a., S. $1-31$.

Feilke, Helmuth (2014a): Argumente für eine Didaktik der Textprozeduren. In: Feilke, Helmuth/ Bachmann, Thomas (Hg.): Werkzeuge des Schreibens. Beiträge zu einer Didaktik der Textprozeduren. Stuttgart, S. 11-34.

Feilke, Helmuth (2014b): Begriff und Bedingungen literaler Kompetenz. In: Feilke, Helmuth/Pohl, Thorsten (Hg.): Schriftlicher Sprachgebrauch - Texte verfassen. (= Deutschunterricht in Theorie und Praxis 4). Baltmannsweiler, S. 33-53.

Feilke, Helmuth/Lehnen, Katrin (2011): Wissenschaftliches Referieren. Positionen wiedergeben und konstruieren. In: Der Deutschunterricht 2011, 5, S. 34-44.

Fix, Martin (2000): Textrevisionen in der Schule. Prozessorientierte Schreibdidaktik zwischen Instruktion und Selbststeuerung. Empirische Untersuchungen in achten Klassen. Baltmannsweiler.

Fleck, Ludwik (1980): Entstehung und Entwicklung einer wissenschaftlichen Tatsache. Einführung in die Lehre vom Denkstil und Denkkollektiv. Frankfurt a.M. [Originalausg. Basel 1935.]

Flower, Linda/Long, Eleonore/Higgins, Loraine (2000): Learning to rival. A literate practice for intercultural inquiry. (= Rhetoric, Knowledge, and Society). Mahwah u.a.

Goody, Jack/Watt, lan/Gough, Kathleen (1986): Entstehung und Folgen der Schriftkultur. (= Suhrkamp-Taschenbuch Wissenschaft 600). Frankfurt a.M.

Gloning, Thomas (2003): Organisation und Entwicklung historischer Wortschätze. Lexikologische Konzeption und exemplarische Untersuchungen zum deutschen Wortschatz um 1600. (= Reihe Germanistische Linguistik 242). Tübingen.

Günther, Hartmut (1995): Die Schrift als Modell der Lautsprache. In: Osnabrücker Beiträge zur Sprachtheorie 51, S. $15-33$.

Häcki-Buhofer, Annelies (2000): Mediale Voraussetzungen: Bedingungen von Schriftlichkeit allgemein. In: Brinker, Klaus et al. (Hg.): Text- und Gesprächslinguistik. Ein internationales Handbuch zeitgenössischer Forschung. 1. Halbbd. (= Handbücher zur Sprach- und Kommunikationswissenschaft 16). Berlin/New York, S. 251-261.

Hyland, Ken (1999): Academic attribution: Citation and the construction of disciplinary knowledge. In: Applied Linguistics 20, S. 341-367.

Illich, Ivan (1991): Im Weinberg des Textes. Als das Schriftbild der Moderne entstand. Frankfurt a.M. Jakobs, Eva-Maria (1999): Textvernetzung in den Wissenschaften. Zitat und Verweis als Ergebnis rezeptiven, reproduktiven und produktiven Handelns. (= Reihe Germanistische Linguistik 210). Tübingen.

Keller, Rudi (1990): Sprachwandel. Von der unsichtbaren Hand in der Sprache. Tübingen.

Kretzenbacher, Heinz L. (1997): Fachsprache als Wissenschaftssprache. In: Hoffmann, Lothar/ Kalverkämper, Hartwig/Wiegand, Herbert Ernst (Hg.): Fachsprachen. Ein internationales Handbuch zur Fachsprachenforschung und Terminologiewissenschaft. 1. Halbbd. (= Handbücher zur Sprach- und Kommunikationswissenschaft 14.1). Berlin/New York, S. 133-142.

Lüling, Britta (2014): Ko-Autorschaft, Schreibkonferenz und individuelles Schreiben. Zur Effektivität schreibdidaktischer Methoden. Staatsexamensarbeit Gießen.

Maas, Utz (2000): Orthographie. Materialien zu einem erklärenden Handbuch zur Rechtschreibung des Deutschen. Osnabrück.

Morek, Miriam/Heller, Vivien (2012): Bildungssprache: Kommunikative, epistemische, soziale und interaktive Aspekte ihres Gebrauchs. In: Zeitschrift für Angewandte Linguistik 57, S. 67-101. 
Morais, Josê (1987): Segmental analysis of speech and its relation to reading ability. In: Annals of Dislexia 37, S. 126-141.

Olson, David R./Astington, Janet Wilde (1993): Cultural learning and educational process. Kommentar zu Tomasello, Michael/Kruger, Ann Cale/Ratner, Hilary Horn 1993: Cultural learning. In: Behavioral and Brain Sciences 16, S. 531-532.

Ong, Walter (1987): Oralität und Literalität. Die Technologisierung des Wortes. Opladen.

Parkes, Malcolm (1999): Klösterliche Lektürepraktiken im Hochmittelalter. In: Chartier, Roger/ Cavallo, Guglielmo (Hg.): Die Welt des Lesens. Von der Schriftrolle zum Bildschirm. Frankfurt a.M./New York, S. 135-153.

Pohl, Thorsten (2007): Studien zur Ontogenese wissenschaftlichen Schreibens. (= Reihe Germanistische Linguistik 271). Tübingen.

Reckwitz, Andreas (2003): Grundelemente einer Theorie sozialer Praktiken. Eine sozialtheoretische Perspektive. In: Zeitschrift für Soziologie 32, S. 282-301.

Reckwitz, Andreas (2006): Das hybride Subjekt. Eine Theorie der Subjektkulturen von der Bürgerlichen Moderne zur Postmoderne. Weilerswist.

Saenger, Paul (1999): Lesen im Spätmittelalter. In: Chartier, Roger/Cavallo, Guglielmo (Hg.): Die Welt des Lesens. Von der Schriftrolle zum Bildschirm. Frankfurt a.M./New York, S. 181-217.

Schleppegrell, Mary J. (2010): The language of schooling. A functional linguistics perspective. New York. [Digital print]. [Erstausg. New York 2004.]

Schmidt, Robert (2008): Stumme Weitergabe. Zur Praxeologie sozialisatorischer Vermittlungsprozesse. In: Zeitschrift für Soziologie der Erziehung und Sozialisation 37, S. 121-136.

Schmidt, Robert (2012): Soziologie der Praktiken. Konzeptionelle Studien und empirische Analysen. Frankfurt a.M.

Schnitzler, Carola Dorothé (2008): Phonologische Bewusstheit und Schriftspracherwerb. (= Forum Logopädie). Stuttgart/New York.

Scribner, Sylvia/Cole, Michael (1978): Literacy without schooling: Testing for intellectual effects. In: Harvard Educational Review 48, S. 448-461.

Scribner, Sylvia/Cole, Michael (1981): The psychology of literacy. Cambridge.

Silverstein, Michael (1979): Language structure and linguistic ideology. In: Clyne, Paul R./Hanks, William F./Hofbauer, Carol L. (Hg.): The elements: A parasession on linguistic units and levels. Chicago, S. 193-247.

Steinhoff, Torsten (2007): Wissenschaftliche Textkompetenz. Sprachgebrauch und Schreibentwicklung in wissenschaftlichen Texten von Studenten und Experten. Tübingen.

Steinseifer, Martin (i.Vorb.): Zitatrahmenkonstruktionen und die Struktur eristischen Argumentierens. In: Feilke, Helmuth/Lehnen, Katrin/Steinseifer, Martin (Hg.): Eristische Literalität.

Sterponi, Laura/Lai, Paul F. (2013): Literacy. In: Oxford Bibliographies in Anthropology. Published online September 2013. Hrsg. v. John Jackson. Internet: www.oxfordbibliographies.com/ view/document/obo-9780199766567/obo-9780199766567-0123.xml;jsessionid=1C0BA1 497C1E34318E1CA9A798D64917 (Stand: 28.1.2015).

Stetter, Christian (1997): Schrift und Sprache. Frankfurt a.M.

Street, Brian (2001): Literacy and development. Ethnographic perspectives. London.

Street, Brian (2009): Ethnography of writing and reading. In: Olson, David R./Torrance, Nancy (Hg.): The Cambridge handbook of literacy. Cambridge u.a., S. 329-345.

Tomasello, Michael/Kruger, Ann Cale/Ratner, Hilary Horn (1993): Cultural learning. In: Behavioral and Brain Sciences 16, S. 495-511; anschließende Diskussion, S. 512-552.

Wenger, Etienne (1998): Communities of practice: Learning, meaning, and identity. Cambridge. 